\title{
CRESCERE SULLA FRONTIERA: DAL TRAUMA ALLA MEMORIA IN BAMBINI E ADOLESCENTI MIGRANTI
}

Virginia De Micco*

\begin{abstract}
Attraverso una serie di incontri con bambini migranti vengono esaminate le specifiche difficoltà incontrate nella ricostruzione del loro senso di identità e di appartenenza. Viene dedicata una particolare attenzione all'analisi della memoria dei traumi connessi con l'esperienza migratoria in cui si manifestano particolarmente conflitti e disconoscimenti per questi bambini.
\end{abstract}

Parole chiave: migrazione, memoria, trauma.

Crescere sulla frontiera significa crescere in una sorta di sospensione del tempo, di fermo-immagine del momento dell'attraversamento di una soglia: i bambini migranti sono immersi in una dimensione di passaggio in cui memoria e oblio continuamente si confrontano. Nel loro caso la 'frontiera' non è soltanto una striscia sottile ma un'intera dimensione in cui forzatamente 'abitare' per un tempo anche lungo.

Ho incontrato i bambini e i ragazzi cui si ispira questo lavoro nell'ambito di una ricerca presso un centro di accoglienza per minori immigrati, situato in Italia, in una zona particolare della provincia di Caserta, nel comune di Castel Volturno. Si tratta di un'area che dopo aver conosciuto un discreto sviluppo economico fino agli anni ottanta è poi andata incontro ad un rapido declino ed attualmente viene privilegiata dai flussi migratori principalmente per gli scarsi controlli statali e dunque per le ampie opportunità di accoglienza degli immigrati irregolari che offre. E' diventata negli anni una sorta di "terra di nessuno", occupata progressivamente da gruppi marginali 'allontanati' da

\footnotetext{
* Società Psicoanalitica Italiana - International Psychoanalytical Association. Caserta/Italia.
} 
altri spazi sociali. II centro è un prodotto spontaneo del territorio che nasce proprio sull'onda dei primi arrivi di immigrati offrendo soprattutto assistenza nella gestione dei bisogni di famiglie e minori. Attualmente ospita sia minori in regime residenziale, soprattutto minori non accompagnati o in affidamento giudiziale, prevalentemente provenienti dall'est Europa o di etnia Rom, sia in regime di semiresidenzialità, si tratta di bambini le cui famiglie sono presenti sul territorio ma che sono seguiti presso il centro per tutte le attività scolastiche, ricreative e assistenziali, prevalentemente di origine africana, provenienti soprattutto da Ghana e Nigeria.

La ricerca si è svolta nell'arco di circa due anni, con una frequentazione costante del centro, in cui, accanto agli incontri con operatori, ospiti e loro familiari, si è inteso condividere lo spazio quotidiano di bambini e ragazzi immigrati, con i quali poi sono state svolte interviste approfondite. Accanto alle modalità dell'inchiesta etnografica, utilizzate per descrivere le caratteristiche socioantropologiche del territorio e del funzionamento del centro, è stata privilegiata negli incontri coi giovani migranti una attitudine di raccolta e di interpretazione dei dati che coniugasse le modalità dell'incontro etnografico e del colloquio psicoanalitico. Non si è trattato dunque di colloqui strettamente clinici, chiesti per qualche manifesto disturbo dei bambini, ma di incontri in cui, seguendo alcune indicazioni del noto psicoanalista Donald Winnicott, si intendeva lavorare su aspetti psichici profondi anche al di fuori di autentici setting terapeutici. Gli incontri si proponevano di sondare l'intreccio singolare tra identità e memoria che veniva a delinearsi nell'esperienza di questi bambini, prestando particolare attenzione al modo in cui erano capaci di raccontare la propria storia: autorappresentarsi in termini 'storici' implicava infatti una domanda sulla propria origine ed appartenenza. Tale costitutiva interrogazione sulla propria origine apre un percorso attraverso il quale si possa diventare ciò che si è, in un movimento, per così dire, di soggettivizzazione della propria origine, per il quale si è letteralmente chiamati ad identificarsi col proprio corpo, col proprio nome, con i propri 'antenati'. Proprio lungo questi versanti i bambini immigrati incontrano, com’è intuibile, particolari difficoltà che possono essere osservate in profondità, al di sotto di superficiali livelli di buona 'integrazione', proprio attraverso strumenti relazionali e concettuali capaci di coniugare ascolto psicoanalitico e sguardo antropologico.

La questione della migrazione chiama inevitabilmente ed immediatamente in causa quella della dimensione costitutiva della cultura per la psiche individuale: della intelaiatura culturale che regge l'apparato psichico. L'essere umano è destinato alla socialità dalla sua stessa biologia 
in quanto necessita di un lungo periodo di accudimento in cui è solo grazie alla relazione con un altro essere umano che può sopravvivere e strutturarsi psichicamente. E necessita di un apparato segnico-simbolico, un apparato culturale dunque, entro e attraverso il quale possa trovare strumenti di rappresentazione emotiva e simbolica per costruire la propria interiorità. L'essere umano è dunque immediatamente immerso in una relazione ed in una cultura, così come è emerso dalla specificità delle sue relazioni costitutive e dalla particolarità delle sue determinanti culturali.

Già Leon e Rebecca Grinberg', psicoanalisti argentini, nel loro pionieristico, e per certi versi ancora insuperato, lavoro sulla Psicoanalisi della migrazione e dell'esilio sottolineavano come l'emigrazione costituisca una vera e propria esperienza di rinascita, con il rischio che l'impossibilità di ri-affiliarsi al nuovo universo culturale in cui si è immersi comporti una insanabile ferita dell'involucro psichico. Rischio tanto più presente per i bambini migranti che si trovano impegnati in una sorta di doppio transito, di doppio passaggio identitario: I'essere bambini mentre si emigra e l'essere migranti mentre si è bambini. Transito psicoantropologico da una parte, che comporta crescita ed evoluzione psichica, la quale deve necessariamente 'appoggiarsi' su un tessuto simbolico e relazionale, ovverosia su un tessuto culturale, per potersi svolgere in maniera efficace, e transito antropopsicologico dall'altra, ovverosia capacità di passare attraverso diverse ricomposizioni del senso di sé e del senso di appartenenza che, per potersi realizzare, necessitano di una struttura psichica capace di contenerle. La condizione specifica dei giovani immigrati è stata spesso interpretata e studiata come assolutamente 'solidale' con quella delle loro famiglie: da un lato si riteneva che i bambini venissero protetti dall'impatto più destrutturate dell'esperienza migratoria sul piano psicologico grazie all'involucro protettivo rappresentato dal contenitore familiare, dall'altro che un'autonoma capacità di socializzazione facesse sentire i suoi effetti piuttosto tardi, prevalendo prima i meccanismi di trasmissione e riproduzione culturale all'interno delle famiglie e delle comunità di origine. Attualmente però è proprio il doppio impatto di due fenomeni intimamente legati ai processi di globalizzazione, come evidenzia Appadurai ${ }^{2}$, a rendere le cose più complesse: gli effetti delle comunicazioni di massa da un lato, la progressiva deterritorializzazione delle comunità e degli individui dall'altro, fanno sì che i contenitori familiari non riescano più ad incapsulare l'esperienza dei bambini migranti e che questi ultimi si trovino a dover fronteggiare molto più rapidamente il compito di costruire il

\footnotetext{
${ }^{1}$ GRINBERG, León; GRINBERG, Rebeca. Psicoanalisi dell'emigrazione e dell'esilio.

${ }^{2}$ APPADURAI, Arjun. Modernità in polvere.
} 
proprio senso di identità e di appartenenza lungo frontiere culturali incerte e instabili.

Quando I'ordine simbolico dei significati vacilla, come accade nell'esperienza migratoria, non si può più essere sicuri dell' 'ovvietà' del mondo, che diventa 'straniero', diventa un enigma irresolubile con gli strumenti mentali che si hanno a disposizione.

\section{Dal trauma alla memoria}

La questione centrale dell'appartenenza si declina non solo sul versante dell'identità ma anche su quello della memoria. Quest'ultimo implica immediatamente la necessità di dipanare il nodo traumatico connesso con l'esperienza migratoria. Innanzi tutto occorre chiarire cosa si possa intendere per 'trauma culturale' connesso con la migrazione. La possibilità di costruire una memoria individuale del transito migratorio, infatti, passa attraverso la necessità di trovare strumenti di rappresentabilità culturale e psichica del proprio 'trauma' migratorio: senza una qualche forma di rappresentazione/ elaborazione dello specifico elemento traumatico connesso alla migrazione non si può accedere alla costituzione di una 'memoria' dell'evento stesso. Solo attraverso questo snodo cruciale può avvenire un 'passaggio' della frontiera che sia anche un 'passaggio' psichico e non una 'frattura' e dunque un crollo psichico irrimediabile.

Necessità di ricordare e necessità di dimenticare si rincorrono e si confrontano nell'esperienza dei bambini migranti. Esperienza in cui riveste un ruolo fondamentale anche la trasmissione psichica attraverso le generazioni, mediante la quale instaurare una catena di memorie individuali e collettive in cui si possa trovare il proprio 'posto' e il proprio 'senso'.

L'affiliazione ad un nuovo ordine simbolico, richiesta dall'esperienza migratoria soprattutto ai giovani migranti, necessita di un tempo in cui sia possibile svolgere quell'imponente lavorio psichico e culturale richiesto da una vera e propria ridefinizione della propria identità.

Alcuni studiosi, tra i quali ad esempio Michele Risso ${ }^{3}$, hanno sottolineato come si possa descrivere l'intero processo di adattamento ad un nuovo contesto culturale nei termini di un microtraumatismo quotidiano e di un processo conflittuale permanente. Da un punto di vista strettamente psicologico, bisogna specificare come in tal modo ci si sposti da una visione del trauma come evento unico caratterizzato dalla sua 'violenza' per la

3 Cf. RISSO, Michele e BOEKER, Wolfgang. Sortilegio e delirio. Psicopatologia delle migrazioni in prospettiva transculturale. 
struttura psichica ad una visione del trauma come serie di eventi, ispirata dal concetto di trauma cumulativo secondo Masud Khan. Questi ultimi progressivamente 'trasformano' la struttura psichica, rendendola magari più vulnerabile e aumentando il rischio che si possa sviluppare una malattia psichica. Da un lato si vede come in tal modo si apra al superamento dell'idea della vulnerabilità psichica costituzionale, in quanto il trauma cumulato negli anni può trasformare la struttura psichica stessa. Dall'altro non è semplice articolare in termini psicologici l'idea di una serie traumatica che cumuli i suoi effetti nel tempo al di fuori del periodo di formazione delle strutture psichiche, ovverosia al di fuori della prima infanzia. Si consideri inoltre che lo stesso concetto di trauma cumulativo in psicologia trae il suo senso solo dalla stretta dipendenza tra madre e bambino nella primissima infanzia, ovverosia risulta concepibile solo all'interno di una matrice relazionale primaria che organizza il mondo esterno ed il mondo interno dandogli significato. Ora è proprio questo che mi sembra estremamente interessante: per poter proporre l'esperienza migratoria come fonte di 'traumaticità' in termini fondati dal punto di vista psicologico occorre riconoscerne lo specifico valore di ricostituzione simbolica e cultural.

In questo senso l'esperienza migratoria corrisponde ad una risignificazione del mondo, delle relazioni e di sé stessi; essa comporta una vera e propria reiscrizione di sé stessi nel mondo e del mondo in sé stessi: richiede dunque dei veri e propri atti di rifondazione simbolica di sé e del mondo. Vorrei sottolineare come è proprio la quotidianità che diventa allora fondamentale fonte di traumaticità perché diventa squisitamente enigmatica: è il significato da attribuire alla realtà che risulta instabile. $\mathrm{E}^{\prime}$ esattamente in questo livello antropologico che si colloca la fonte effettiva e la pensabilità stessa del trauma individuale, del vulnus psicologico. In fondo è come se non ci si potesse più fidare del mondo, che diventa 'straniero' appunto.

Trauma dunque che manifesta i suoi effetti sul piano psichico ma che si impianta in una specifica attitudine alla costruzione simbolica del proprio mondo e di sé stessi, al di fuori della fondamentale costititutività dell'esperienza culturale non è dunque pensabile l'idea di un trauma connesso alla migrazione: esso può essere psicologico solo in quanto è simultaneamente un trauma culturale. Pertanto può essere curato e compreso soltanto tenendo costantemente presente questo doppio piano di riferimento.

Un altro elemento centrale da tenere presente, direttamente implicato dalle riflessioni precedenti, riguarda l'apparente invisibilità del trauma così inteso, ovverosia il suo non manifestarsi immediatamente. Questo manifestarsi non riguarda solo una eventuale ricaduta in termini clinici o latamente psicologici, legati cioè ad un generico disagio individuale o relazionale, ma 
riguarda più specificamente i modi di costruzione e rappresentazione della propria esperienza, investe in pieno cioè la modalità in cui i migranti stessi percepiscono e rappresentano la loro esperienza. Diversi studiosi, tra i quali Rosoli $^{4}$ e Sayad ${ }^{5}$, hanno infatti notato come la migrazione mostri spesso la sua "vera natura" a posteriori, come in una sorta di retrospettiva, mentre Raison $^{6}$ sottolinea addirittura che gli effetti generali dell'emigrazione su una popolazione "possono farsi sentire a lungo termine, sulla scala di una o due generazioni". Sayad7, ad esempio, rileva come molti dei lavoratori immigrati da lui incontrati riuscissero a raccontare la loro storia, e dunque a rappresentare a sé stessi tutta la difficoltà e la delusione della loro esperienza, tutta la durezza di ciò che loro stessi avevano vissuto, soltanto sul finire della loro vita lavorativa. Parafrasando i termini del noto psicoanalista inglese Donald Winnicott ${ }^{8}$, ed adattandoli al nostro contesto, si potrebbe dire che solo alla fine della loro vita riuscivano a vivere ciò che gli era accaduto, riuscivano cioè a costruire degli strumenti simbolici attraverso i quali rappresentarsi e dare significato al proprio vissuto, traducendolo così da evento in esperienza vissuta e in memorie trasmissibili. Nel far ciò sono proprio gli elementi traumatici a dover trovare una loro visibilità ed una loro rappresentabilità. Paradossalmente è come se senza riconoscimento del trauma non fosse possibile costruire memoria. Ancora una volta sarà importante sottolineare come è proprio I'intervento degli strumenti simbolici come il racconto, nella specifica forma della rievocazione della propria esperienza, che consentono di dare forma, ovverosia contemporaneamente visibilità e rappresentabilità, al vissuto.

In questo senso potremmo dire che sul piano culturale il trauma, piuttosto che indicare semplicemente una rottura di continuità sul piano delle rappresentazioni condivise (il cosiddetto 'shock culturale'), riguarda il travaglio simbolico relativo al senso da attribuire all'esperienza. Se questo è vero per la quotidianità, come abbiamo visto, lo è in misura ancora maggiore rispetto ad alcuni eventi critici. Non a caso parlo di eventi critici e non di 'traumi', perché è come se tali 'eventi' non fossero ancora riusciti a diventare traumi.

Nelle storie dei nostri piccoli protagonisti ci sono spesso eventi critici: morte o abbandono da parte di genitori, violenze fisiche e psicologiche subite, separazione prolungata e violenta dalle famiglie. Colpisce però come tali avvenimenti siano spesso singolarmente assenti dai loro racconti, come

\footnotetext{
${ }^{4}$ ROSOLI, Gianfausto. La condizione sanitaria dell'emigrante.

${ }^{5}$ SAYAD, Abdelmalek. La doppia assenza.

${ }^{6}$ RAISON, Jean-Paul. Migrazione.

7 SAYAD, op. cit.

${ }^{8}$ WINNICOTT, Donald. La funzione di specchio della madre e della famiglia nello sviluppo infantile.
} 
non riescano a trovare una collocazione nella rappresentazione di sé, sembra proprio che siano avvolti in un travaglio simbolico in cui tali eventi sono, per così dire, 'in attesa di sapere' cosa devono o possono rappresentare per il soggetto, per quella costruzione culturale individuale che è il soggetto, come sottolinea Wieviorka. In questa operazione di certo sono coinvolte le modalità di trasmissione culturale transgenerazionale che nei casi esaminati non sembrano funzionare in maniera efficace. I nostri piccoli protagonisti sembrano, in questo senso, incapaci di attribuire un corretto senso culturale agli avvenimenti critici che li hanno colpiti e si comportano quasi come se fossero accaduti solo presso di loro e non dentro di loro. Indizi di dolorosi enigmi irrisolti che devono essere 'opacizzati', piuttosto che svelati.

Da questo punto di vista allora bisogna guardare al trauma non più come ad un evento, ma come a un processo. L'evento critico può diventare esperienza traumatica nel momento in cui avviene una sua elaborazione simbolica, nel momento in cui può assumere una configurazione culturale: può essere detto, ricordato, utilizzato per costruire memoria individuale e collettiva.

Fino a quando questa operazione non sarà possibile, fino a quando non ci sarà posto per la 'costruzione' culturale del trauma, quello che si rileverà saranno piuttosto le attive strategie di cancellazione e di occultamento poste in atto, quella singolare 'opacità' delle storie dei migranti che Sayad invitava a rispettare: lasciare ai migranti "I'opacità delle loro storie", estrema e paradossale forma di resistenza. "L'opacità di un linguaggio che non si apre fin dal primo istante" scrive "è senza dubbio l'informazione più importante e più rara" 9 .

\section{Le forme dell'oblio}

Spostare l'attenzione verso le "forme dell'oblio", come le chiama Marc Augé $^{10}$, sarà allora una delle opzioni perseguibili, che cercherà di capire la forma di certi silenzi e di certe omissioni, veri e propri depositi di significato.

Certo colpisce la grande difficoltà manifestata da bambini e ragazzi nell' organizzare una narrazione relativa alla propria esperienza, ovverosia nel dipanarla lungo l'asse temporale: il prima e il dopo che si delineano hanno poco a che fare con un andamento cronologico e riflettono piuttosto improvvise fratture della loro esistenza, anzi la stessa giustapposizione di universi che appaiono così distanti tra di loro (il prima e il dopo appunto,

\footnotetext{
9 SAYAD, op. cit., p. 17.

10 AUGÉ, Marc. Le forme dell'oblio. Dimenticare per vivere.
} 
che simultameamente sono anche un qui e un altrove) accentua questo carattere di discontinuità piuttosto che di continuità. Se una delle caratteristiche principali della narrazione autobiografica consiste proprio nel restituire/ricostruire la continuità dell'esperienza, nel nostro caso invece la preoccupazione principale appare piuttosto legata alla necessità di occultare qualcosa, di dimenticare piuttosto che di ricordare. La necessità assoluta di dimenticare per continuare a vivere è un tema ricorrente nelle storie di migranti, essa risponde sia a una necessità psichica individuale che a una 'richiesta' sociale, esercitata 'implicitamente' dal contesto di immigrazione.

L'azione sociale, il suo modellamento dell'esperienza dei singoli è qui rintracciabile in ciò che in un certo senso impone di dimenticare. La "violenza simbolica", come la chiama Pierre Bourdieu", si esercita qui esattamente in una sorta di complicità tra necessità individuale di sopravvivere psichicamente (l'oblio come forma di resistenza, il "dimenticare per vivere", come sottolinea Augè) e implicita sottomissione a un forma di vincolo sociale e simbolico che impone di configurare la propria vita in una determinata maniera. La costruzione della memoria individuale, la possibilità cioè di mettere il proprio presente in una relazione significativa col passato, appare dunque come un terreno ben più accidentato e conflittuale rispetto all'organizzazione della quotidianità, in cui prevale una attitudine mimetica da parte dei bambini e dei ragazzi migranti apparentemente aconflittuale. Nessuno dei bambini o dei ragazzi intervistati infatti ha manifestato, o voluto riconoscere, particolari difficoltà nella gestione della quotidianità, ma le cose diventano decisamente più complicate se si indaga su questa specifica funzione identitaria che è la memoria. La semplice richiesta di ricordare, di mettere in continuità presente e passato, qui e lì, può essere eccessiva per questi bambini: rimettere in gioco il passato significa sì rientrare in contatto con qualcosa che a che fare con la propria origine, con la propria provenienza, ma che assume una sorta di alterità rispetto all'oggi, rispetto al quotidiano, rispetto alla propria percezione di sé. Un passato dunque che nel nostro caso corre il rischio di entrare in rotta di collisione col presente, in quanto portatore del fondamentale enigma sulla propria appartenenza.

Come scrivono infatti Fabietti e Matera ${ }^{12}$ :

In quanto pilastro di qualunque costruzione identitaria, ed essa stessa rappresentazione del passato frutto di una costruzione, la memoria costituisce un oggetto privilegiato dell'antropologia culturale e sociale (...) Ma nel processo di costruzione

\footnotetext{
${ }^{11}$ BOURDIEU, Pierre. Per una teoria della pratica.

${ }^{12}$ FABIETTI, Ugo; MATERA, Vincenzo. Memorie e identità. Simboli e strategie del ricordo.
} 
dell'identità, la memoria ha sempre e comunque lo stesso significato e la stessa funzione: offrire una rappresentazione dotata di senso del proprio presente.(...) La memoria non è quindi una semplice 'registrazione' del passato, ma una rappresentazione di quest'ultimo ottenuta attraverso il ricordo da un lato e l'oblio dall'altro.

Da un lato, dunque, è proprio in funzione della sostenibilità del proprio presente, per conservarne il 'senso', che i bambini 'rimaneggiano' la loro memoria, dall'altro diventa cruciale indagare secondo quali codici di rappresentabilità riorganizzano il loro passato.

Questa operazione non è infatti praticabile per tutti nella medesima maniera, è importante sottolineare a questo proposito quanto possa essere distante, e a tratti addirittura divergente, I'esperienza degli adolescenti emigrati da soli, da quella dei bambini emigrati al seguito delle famiglie. Mentre i primi costituiscono a tutti gli effetti una prima generazione di immigrati, rappresentando una prima fase emigratoria per il loro paese, dunque con una stabilità conservata delle strutture simboliche e culturali delle comunità di provenienza, i secondi invece costituiscono una seconda generazione di cui condividono tutte le problematiche. E' sorprendente vedere in questa sorta di schiacciamento temporale, dal momento che in fondo pochi anni di differenza li separano, sovrapporsi e divergere declinazioni così differenziate dell'esperienza migratoria.

Una delle aree di differenziazione più evidenti è proprio in rapporto alla memoria, sia del proprio passato che del paese di emigrazione. Per i ragazzi di prima generazione si tratta, infatti, di una memoria viva e personale, ma di cui non si parla volentieri, una memoria 'reale', per i bambini di seconda generazione si tratta spesso di una memoria 'occultata'.

Una delle preoccupazioni costanti nelle autopresentazioni dei bambini di seconda generazione, con maggiori o minori accentuazioni individuali, resta infatti quella di dimostrare all'interlocutore che loro non sono 'diversi'. Sembrano voler mostrare all'interlocutore che non c'è proprio nulla da vedere, che la loro vita è ovvia,'normale': la strategia di occultamento non potrebbe essere più completa. In fondo questo tentativo di rendersi identici ai bambini autoctoni può essere considerato come un altro modo per rendersi invisibili allo scopo di rendersi accettabili: ovverosia rendere impercettibile la loro differenza, anche al prezzo di cancellare una parte di sè, al fine di rendere accettabile la loro presenza.

Questa impossibilità di memoria si intreccia con l'invisibilità del trauma che evidenziavamo prima, e con il rischio di fallimento dei meccanismi 
di trasmissione culturale transgenerazionale. Vi è una modalità del tutto particolare in cui tale trasmissione può mantenersi, una sorta di fiume carsico, di trasmissione clandestina, che si realizza quasi all'insaputa di sè stessi. Memoria presente più nei gesti e in una sorta di tacita comunicazione che nelle parole, una sorta di "memoria involontaria", che può riemergere improvvisamente sotto forma di cose che 'non sanno di sapere'. Così è "I'inglese che mi era rimasto appiccicato alla testa, anche se io in casa non I'avevo mai parlato" di cui racconta Willy, un bambino di origine nigeriana, o la "lingua segreta" di Aki, di cui più oltre è riportata la storia. Una sorta di appartenenza criptica e cifrata, che deve rendersi 'irriconoscibile', in un certo senso, per coloro stessi che la 'portano'.

Tali elementi funzionano come dei veri e propri segni cifrati dell'appartenenza, di cui forse solo in seguito sarà possibile riappropriarsi, de-criptarli, in ogni senso, sia nel senso di poterli 'interpretare' cognitivamente sia nel senso di poterli far uscire dalla cripta affettiva in cui necessariamente sono stati sigillati.

In questa situazione il 'centro' di accoglienza diventa invece un attivo organizzatore delle memorie individuali di questi bambini, proprio a causa della difficoltà, spesso insostenibile di mettere in relazione presente e passato, e soprattutto qui e lì, intesi come paese di immigrazione e paese di 'origine', che devono invece spesso restare regioni assolutamente separate dell'esperienza e del ricordo.

Spesso, del resto, una autentica funzione 'terapeutica' in questi casi può essere costituita dall'essere testimoni di una lenta e progressiva possibilità di riappropriazione della memoria.

\section{La storia di Aki}

Alcuni ragazzi, come Aki, sono costretti a rinnegare completamente una parte consistente del loro passato, specificamente della loro appartenenza familiare. Per loro, in maniera ancora più consistente che per gli altri ospiti, il centro di accoglienza in quanto luogo fisico e deposito di affettività costituisce un vero e proprio guscio psichico, un involucro mentale cui aderire integralmente al punto tale da non poter nemmeno immaginare la propria vita al di fuori del centro di accoglienza.

Aki è solo, le sue 'origini' sono avvolte nell'incertezza, non si sa con precisione quanti anni abbia, dice di provenire dalla ex-jugoslavia, "proprio dalla Serbia, dove c'è stata la guerra" ci tiene a precisare, ma non ricorda il nome del suo paese. La sua memoria è confusa e reticente: aveva dei fratelli 
più piccoli, nonni e zii, un padre piuttosto severo sullo sfondo. L'unica figura che emerge con maggiore distinzione è quella della madre.

Ti dico la verità i miei genitori mi hanno venduto, oppure quello, zio, mi ha rapito, non lo so, so solo che se era per mia madre, no, lei non mi avrebbe mai venduto, mi voleva bene, sono sicuro che piange per me; un giorno mi ha detto Aki vai a comprare i pomodori e all'uscita c'era quello, zio, che ha detto Aki vieni, tua madre ha detto di venire con noi, e così mi ha portato in Italia.

A quell'epoca Aki aveva all'incirca dieci anni ma sembra non ricordare nulla con chiarezza, sembra quasi un sogno confuso quello che riferisce, anche la risonanza sentimentale di questi eventi inclina più ad una sorta di rassegnazione che non al rancore o alla drammaticità dell' essere sottratti alla propria famiglia. In questo distacco sembra esserci più una sorta di incredulità, in fondo quasi di irrappresentabilità interiore (si può essere rapiti come si vanno a comprare i pomodori al mercato), che non di violenza. Sembrano ricordi appartenenti ad un'altra vita, la cui verità è stata riscritta tante volte, a seconda delle versioni che di volta in volta potevano ritornare più utili, che Aki stenta ormai a rievocare effettivamente quello che è stato. I ricordi cominciano a farsi più chiari con l'arrivo in Italia e un difficile itinerario fatto di violenze quotidiane, di percosse, di partecipazione obbligata a piccoli furti e scippi. "Se non rubavo abbastanza mi picchiava, guarda non dico bugie una volta mi si è seduto sopra, mi è rimasto lo sterno storto", dice Aki scoprendosi il petto. Aki racconta tutto con una sorta di oggettività, di mancanza di drammaticità.

Per un tempo imprecisato, alcuni mesi forse più di un anno, Aki vive con la famiglia dell'uomo che lo ha rapito o a cui è stato venduto, indicato sempre come lo zio, come spesso questi uomini si fanno chiamare dai loro bambinischiavi, in una ambigua sovrapposizione tra modello e attributi familiari e attitudini di rispetto e sottomissione. Aki non essendosi rivelato troppo bravo nel rubare rimane spesso a casa con la moglie dello zio e i loro bambini più piccoli, dà una mano nelle faccende domestiche, si rende utile, tenta di ricostruire una dimensione domestica la cui mancanza è talmente assoluta da non poter essere avvertita, pensata, ma solo incessantemente riprodotta e ambiguamente colmata (negata da una parte, ambiguamente riparata dall'altra, poiché non è la sua casa che Aki cerca ma una casa qualsiasi a cui adattarsi). Si sente anche ambiguamente legato a questa dimensione familiare nella cui atmosfera vuole continuare a vivere: avrebbe infatti una prima volta l'occasione di fuggire in seguito ad un grave litigio tra lo zio e la moglie, 
durante il quale rimane incustodito, ma non ha il coraggio di lasciare soli i loro figli piccoli: "il bambino piccolo piangeva e io non lo volevo lasciare. Non sapevo cosa fare", racconta Aki. Nel frattempo hanno attraversato tutta I'Italia venendo a stabilirsi nelle campagne del meridione, vivono in un casolare diroccato in cui hanno stabilito il loro quartiere generale. Approfittando di un momento in cui è solo Aki decide di fuggire. $\mathrm{E}^{\prime}$ in questo momento che il suo racconto si fa drammatico e vivo, nel lungo racconto delle peripezie dei giorni di fuga e di angoscia in cui il ristretto spazio di queste campagne si dilata fino a diventare una sorta di far west, lo sfondo di un'avventura estrema irta di pericoli come una savana tropicale. Si getta in un "fiume" (forse un canale per l'irrigazione come ce ne sono molti da queste parti) e trascorre buona parte della notte nell'acqua per non farsi trovare, coi vestiti inzuppati quando ormai si sente più sicuro comincia ad allontanarsi, quasi timidamente, dal 'suo' casolare, quella sorta di casa-prigione, che era comunque un punto di riferimento in un ambiente sconosciuto. Aki sa che l'unico contropotere in grado di proteggerlo dallo zio è rappresentato dalla polizia, si fa indicare il posto di polizia più vicino e chiede aiuto raccontando confusamente la sua storia. Viene quindi affidato al centro di accoglienza, i cui responsabili diventeranno in seguito i suoi tutori legali. Aki ha dovuto raccontare tante volte la sua storia e a tante persone diverse: poliziotti, magistrati, assistenti sociali, professori, che a questo punto la sua stessa storia, e in questo senso la sua memoria, la sua stessa identità, cominciano a subire una serie di rimaneggiamenti legati alla necessità di non lasciarsi sfuggire questa unica possibilità di salvezza. Questa possibilità di effettuare un vero e proprio salto antropologico, di entrare letteralmente in un altro mondo, il mondo delle tutele e delle garanzie, il mondo dell'inclusione, deve essere mantenuta ad ogni costo, anche se questo significherà diventare un'altra persona.

Chi è Aki? Aki è quello che voi volete che sia. Egli cerca costantemente di intuire quale rappresentazione di sé può essere più utile in determinate circostanze. La sua storia sembra diventare la sua unica ricchezza, una sorta di merce di scambio, il cui significato non è più una faccenda individuale ma assume un ruolo pubblico, per così dire, diventa uno spazio di negoziazione e di mediazione col tessuto sociale e con gli interlocutori istituzionali. Essa pertanto viene sottratta alla memoria individuale: è lo stesso Aki che, con modalità in larga parte inconsapevoli, strumentalizza una parte di sé, della 'sua' storia, si aliena dalla 'sua' memoria. Una delle prime ricadute si ha ad esempio sul piano dell'età anagrafica. Infatti alla fatidica soglia dei diciotto anni corrisponde l'uscita dal sistema di tutele previste per i 'minori', è quindi ovvio che si tenda ad abbassarsi l'età pur di continuare a godere di questi benefici. 
Aki ha talmente manipolato questo aspetto di sé che ora, pur continuando a ricordare la sua vera data di nascita, ha smarrito il senso interno della sua età, pur avendo quasi venti anni, si presenta e si comporta come un ragazzino di quindici anni, è diventato un ragazzino di quindici anni e sembra non riuscire a pensarsi al di fuori di questa dimensione. Poiché la memoria si è dovuta piegare alle esigenze del presente anche il futuro non sembra potersi distendere davanti a lui, ma resta come bloccato in un immobile e dilatato presente in cui si hanno sempre quindici anni. Crescere, rientrare in possesso della propria età - che significa anche della propria memoria e della propria storia - è in questo caso troppo pericoloso, sembra comportare il rischio di cadere nuovamente nel mondo dell'esclusione, in quell'altro mondo che scorre così vicino ma così impenetrabile rispetto al 'nostro' mondo. Aki ha poi individuato il casolare dove aveva vissuto con lo zio, è relativamente vicino al centro di accoglienza, nelle campagne circostanti, ma sembra essere talmente distante da appartenere appunto ad un altro mondo, dal quale Aki vuole essere separato e protetto. Anche presso il centro di accoglienza Aki si rende utile, tende a ritagliare per sé un posto privilegiato, appare spesso seccato dalla difficoltà di avere uno spazio tutto suo, una stanza tutta sua. Voltandosi attorno nella stanza in cui si svolge il nostro colloquio, come abbracciandola con lo sguardo, mi dice "questa era la mia stanza poi ho dovuto lasciarla ai bambini più piccoli, ai nuovi che sono arrivati, lì ci tenevo le mie cose". Chiama il responsabile del centro 'papà' ma non riesce a chiamare 'mamma' la moglie di quest'ultimo, comunque la sua mamma è quella che è rimasta a casa, che gli vuole bene da qualche parte del passato.

$\mathrm{E}^{\prime}$ solo a questo punto che emerge il desiderio di radicamento di Aki. Per lui che ha raccontato quasi senza grandi emozioni una storia così drammatica e penosa, quello che è davvero impensabile è potersi allontanare dal centro di accoglienza, la sua sofferenza e la sua rabbia vengono sollecitate solo da questa prospettiva. Il centro è la sua casa, è qui che rivendica la 'sua' stanza, il 'suo' spazio, la sua vita al di fuori di questo spazio protettivo è quasi impensabile. Ha frequentato per alcuni anni la scuola, ma con scarso rendimento. Temendo di perdere la protezione e le tutele giuridiche e sociali cui sentiva che solo le sue difficoltà e le sofferenze patite gli davano accesso, Aki ha enfatizzato i suoi 'handicap', per così dire. Non solo ha dichiarato un'età inferiore a quella reale, ma ha nascosto anche alcune sue capacità, ad esempio non ha mai detto che andava a scuola e quindi sapeva leggere e scrivere nella sua lingua, temendo che questo sarebbe bastato a farlo allontanare dal centro. Ha anche radicalizzato il distacco e la perdita di ricordi relativa al suo paese temendo che potesse essere svolta un'indagine 
più approfondita sul suo passato, sembra essersi reso piccolo e indifeso come un bambino per poter essere accolto come un bambino. Insomma Aki ha attivamente cancellato e amputato una parte di sé, della sua memoria e delle sue capacità, nel timore di perdere la possibilità di sopravvivere come persona: si è dovuto rassegnare a morire in parte per sopravvivere in parte. Nelle sue modalità di comportamento quotidiane incessantemente sembra materializzare questo conflitto: da un lato si mostra molto capace ed indipendente, 'si rende utile', dall'altro manifesta un forte timore che se tali capacità individuali aumentano troppo questo possa significare un'espulsione dal centro, da ciò derivano tutta una serie di fallimenti, altrimenti inspiegabili, sul piano scolastico e lavorativo. Singolarmente per Aki I'unico legame reale ma segreto col passato è rimasto concentrato nella lingua materna, passa intere serate ad ascoltare in cuffia la radio sintonizzata su emittenti serbe, come una sorta di ninna-nanna, di ricordo del paese natale. Si lascia cullare prima di addormentarsi dal suono di questa lingua evidentemente mai dimenticata, proprio quando ostentatamente sembra aver smarrito del tutto il senso della sua appartenenza al suo passato. Eppure nemmeno lui sa dire perché sembra non poter fare a meno di quei suoni familiari, sembra dover andare ogni notte a questo appuntamento segreto e solitario in cui si rifugia un senso di sé che nessuno possa scoprire. Quello che non c'è, che nessuno sa che esiste, non può essere perduto.

Dunque attorno alle frontiere del centro di accoglienza si addensano le possibilità di una riformulazione identitaria di bambini e adolescenti migranti con tutto il rischio, però, che tale processo fallisca e che lo stesso centro possa invece diventare un margine, e che si avvii una progressiva e irreversibile marginalizzazione sul piano sociale. Rischio presente a diversi livelli e con diverse modalità per tutti gli ospiti del centro: per gli adolescenti di prima generazione che non sanno se riusciranno a rientrare nel flusso progressivo della loro vita una volta diventati maggiorenni; per i ragazzi senza famiglia che addirittura sentono come inesistente e impensabile la propria vita al di fuori del centro di accoglienza; per i bambini di seconda generazione, per i quali sembra impossibile al momento fare a meno, sia nella quotidianità che nella stessa rappresentazione di sé, dell'intermediazione simbolica e sociale del centro rispetto al loro stesso mondo.

L'esercizio della memoria diventa allora un gioco estremamente rischioso, in quanto comporta una continua riformulazione del centro e del margine, di sé stessi e del proprio mondo, riproponendo quello che appare come un paradosso costitutivo dell'esperienza dei bambini migranti, per i quali costruire la propria identità passa attraverso il rischio di perderla. 


\section{Bibliografia}

APPADURAI, Arjun. Modernità in polvere. Roma: Meltemi, 2001.

ASSOUN, Paul-Laurent. Freud e le sciene sociali. Psicoanalisi e teoria della cultura.

Roma: Borla, 1999.

AUGÈ, Marc. Storie del presente. Per un'antropologia dei mondi contemporanei. Milano: II Saggiatore, 1997.

. Il senso degli altri. Torino: Boringhieri, 2000.

. Le forme dell'oblio. Dimenticare per vivere. Milano: Il Saggiatore, 2000.

BOURDIEU, Pierre. Per una teoria della pratica. Milano: Cortina, 2003.

DE MICCO, Virginia. Corpi contesi, storie sospese. Percorsi dell'identità e della memoria in bambini immigrati: uno studio psicoantropologico. Koinos. Gruppo e funzione analitica, v. XXVII, n. 1, 2006.

. Growing up on the border. Identity routes in immigrant children. Journal of European Psychoanalysis, v. II, n. 23, 2006.

. Corpi Nomi Storie. Vicissitudini dell'identità in bambini migranti. Rivista italiana di gruppoanalisi, v. XXII, n. 2, 2008.

FABIETTI, Ugo; MATERA, Vincenzo. Memorie e identità. Simboli e strategie del ricordo. Roma: Meltemi, 1999.

FREUD, Sigmund. Psicologia delle masse e analisi dell'lo. OSF, v. 9, 1921.

FRIGESSI CASTELNUOVO, Delia; RISSO, Michele. A mezza parete. Emigrazione nostalgia malattia mentale. Torino: Einaudi, 1982.

GLISSANT, Édouard. Poetica del diverso. Roma: Meltemi, 1998.

GRINBERG, León; GRINBERG, Rebeca. Psicoanalisi dell'emigrazione e dell'esilio. Milano: Angeli, 1990.

NATHAN, Tobie. La follia degli altri. Etnopsichiatria. Firenze: Ponte alle grazie, 1990.

RAISON, Jean-Paul. Migrazione. In Enciclopedia, v. 9, Torino: Einaudi, 1978, p. 258-311.

REMOTTI, Francesco. Contro l'identità. Bari: Laterza, 1996.

RISSO, Michele; BÖKER, Wolfgang. Sortilegio e delirio. Psicopatologia delle migrazioni in prospettiva transculturale (a cura di Lanternari, Vittorio; De Micco, Virginia; Cardamone, Giuseppe). Napoli: Liguori (I ediz. 1992), 2000.

ROSOLI, Gianfausto. La condizione sanitaria dell'emigrante. In Atti del I Convegno internazionale Medicina e Migrazioni. Roma, 9-10 aprile 1988.

SAYAD, Abdelmalek. La doppia assenza. Milano: Cortina, 2002.

WINNICOTT, Donald. La funzione di specchio della madre e della famiglia nello sviluppo infantile. In Gioco e realtà. Roma: Armando, 1974. 


\section{Abstract}

Growing up on the frontier: from trauma to memory of the migrant children and adolescents

A series of meetings with migrant children reveals specific difficulties in rebuilding their sense of identity and belonging. Particular attention is given to the analysis of the memory of the traumas associated with the migratory experience that caused the children conflicts and disownments.

Keywords: migration, memory, trauma.

Articolo ricevuto il 25/02/2014. Accettato per la pubblicazione il 23/05/2014. Received for publication on February, 25 Accepted for publication on May, 23 ${ }^{\text {th }}, 2014$. 